\title{
LARGE-SCALE MIMO MC-CDMA SYSTEM USING COMBINED MULTIPLE BEAMFORMING AND SPATIAL MULTIPLEXING
}

\author{
Nguyen Huu Trung*, Doan Thanh Binh \\ Hanoi University of Science and Technology, 1 Dai Co Viet, Hai Ba Trung, Hanoi \\ *Email: trung.nguyenhuu@hust.edu.vn
}

Received: 7 February 2017; Accepted for publication: 4 December 2017

\begin{abstract}
This paper proposes a novel Large-scale (massive) Multi-input Multi-output Multicarrier Code division multiple access (LS MIMO MC-CDMA) model and application to the Fifth-Generation Mobile Communication Systems (5G). This system uses combined cylindrical array antenna multiple beamforming architecture with spatial multiplexing. The model is optimized by Min-Max criteria in order to minimize side lobes and maximize the reduction of propagation loss. The Monte Carlo simulation results unify with the analytical solution for system performance.
\end{abstract}

Keywords: large-scale/massive MIMO, MC-CDMA, multiple-beamforming, Reduced-Rank, array antenna, spatial multiplexing.

Classification numbers: 4.2.3, 4.3.3.

\section{INTRODUCTION}

Due to the explosive growth of smartphones, portable devices and digital content, data traffic has grown exponentially. Therefore, to meet these demands, the $5^{\text {th }}$ generation mobile communication system (5G) has been expected to support super-fast speed and super-high capacity. The user data rate is 1000 times higher compared to current systems (LTE/LTE-A), of tens of Gigabits per second [1]. Besides, this system also has to improve the mobility robustness of mobile applications, and support machine to machine (M2M), human to machine (M2H) interfaces [2].

There are many different frequency schemes for 5G system [3]. 5G system can use overlapping bands on the current UHF band $(0.3 \div 3 \mathrm{GHz})$ in macro-cell configuration and use low SHF band (Super High Frequency) ranging $3 \div 6 \mathrm{GHz}$, high SHF ranging $6 \div 30 \mathrm{GHz}$ with small-cell configuration. To achieve transmission rate at tens of Gigabit per second, the frequency range has to be hundreds of $\mathrm{MHz}$. If planar array antenna is configured at $20 \mathrm{GHz}$ frequency band and the distance between the elements is set at a half wavelength in length (7.5 $\mathrm{mm}$ ), the size of the array antenna having 256 elements will be $12 \mathrm{~cm}^{2}$. This is the actual size may be applied and so, some elements may increase as we increase the frequency. However, the higher frequency is, the greater transmission loss is [4].

Multicarrier - Code division multiple access (MC-CDMA) is a combination of CDMA technique and multicarrier modulation to meet the requirements about transmission speed, robustness and frequency selective fading combat, effective bandwidth usage for 5G [5]. 
Additionally, MIMO systems use multiple antennas architecture in both the transmitter and the receiver, add spatial dimensions, increase degrees of freedom of the system, exploit diversity gain, increase speed as well as the system capacity. As a result, MIMO MC-CDMA systems exploit beneficially space, frequency and code diversities [6]. The increase in capacity based on MIMO techniques for user data in downlink (one point to multiple points) uses spatial multiplexing.

In order to achieve a high data rate, the spatial multiplexing can be combined with beamforming methods. Various schemes for spatial multiplexing systems have been proposed [7]. Channel capacity maximization can be achieved by the water-filling solution using the SVD (Singular Value Decomposition) of the channel matrix [8], or other channel matrix precoding design techniques [8], [9]. However, the channel state information feedback could be difficult in high data rate communication systems. So that, to achieve high data rate, the main beam must be narrow, and side lobes must be low.

In this paper, we propose a large scale MIMO MC-CDMA system model for the next generation mobile communication systems by using cylindrical array antennas combining with multiple beamforming precoding. Compared to the previous results in the open literature, the new contributions of our study are as follows. Firstly, the proposed model is optimized by MinMax criteria in order to minimize side lobes and maximize desired signal while minimizing interferences. Secondly, we apply reduce-rank model in order to obtain the optimal beamforming performance with low computational complexity.

The rest of the paper is organized as follows. In the next section, the proposed system model is introduced while in the section III, we present multiuser detection applied to this system. Section IV presents simulation results. Concluding remarks and directions for further researches are mentioned in the last section.

\section{SYSTEM MODEL}

Multicarrier - Code division multiple access (MC-CDMA) has two forms: The first one is Multicarrier direct sequence Code division multiple access (MC-DS-CDMA) and the second one is MC-CDMA [1]. For the case of MC-DS-CDMA system, the data symbols on subcarriers are spread over time by multiplying the chips on a spreading code by the data symbol on the subcarrier. Meanwhile, MC-CDMA spreads each user symbol in the frequency domain, that is, each user symbol is carried over multiple parallel subcarriers, but it is phase shifted according to a code value. This system uses OFDM to modulate spreading codes and chips. These two systems are potential candidates for the next generation systems, especially 5G systems [10].

\subsection{Signal and the transmitter model}

Let us consider large scale/massive MIMO MC-CDMA at a base station serving $N_{M S}$ users illustrated in Figure 1. The base station uses $N_{T}$ transmit antennas and each user uses $N_{R}$ receive antennas. We assume $N_{T} \gg 1$ for a large-scale/massive MIMO system. Assume that channel state information feedback is ideal, a MIMO channel which is established by $N_{T}$ transmit antennas and $N_{R}$ receive antennas is divided into $N_{C}$ independent sub channels spatially with $N_{C}=\min \left\{N_{T}, N_{R}\right\}$. User data can be transmitted over sub channels because each sub channel can experience different channel conditions due to fading effect and multipath at different frequencies with different SNR ratios. The number of the spatial channels is determined by the 
number of modes of the MIMO channel and depends on channel response matrix between $N_{T}$ transmit antennas and $N_{R}$ receive antennas [11].

Let $\mathbf{d}_{u}=\left\{d^{(u)}[i]\right\}$ denote binary input data of user $u$ at time instant $i$. Suppose that $d^{(u)}[i]$ takes values \pm 1 with equal probability and bit cycle $T_{b}$. The input data is channel encoded, scrambled to assure that the noise affecting each symbol is independent, then fed through symbol mapping module (using modulation schemes such as BPSK, QPSK, QAM,...). After that, we have complex modulation symbols having symbol cycle $T_{S}=n T_{b}$ and modulation level $M=2^{n}$.

The MIMO pre-coding process by singular value decomposition (SVD) transform is performed at the transmitter to determine the eigenmode of the MIMO channel and the set of beamforming vectors. On the basis of eigenmodes, it determines the power distribution over orthogonal symbol streams, thereby determines the speed, modulation method, coding schemes corresponding to the power level distributed optimally. Not only speed data modulation but also coding method can be adapted according to eigenmodes. Channel estimation is accomplished through pilot data multiplexed in time (TDM) or code (CDM) on all or a subset of the transmitted user data streams [12].

The power distribution to symbol streams is usually performed by water filling algorithm. Data streams having separate modes with high SNR will take precedence. After the distribution, we have a complex symbol vector $N_{T}-1$ elements $\mathbf{x}_{u}=\left[x_{0}^{(u)}, x_{1}^{(u)}, \ldots, x_{N_{T}-1}^{(u)}\right]$ formed from the precoding matrix $\mathbf{P}_{u}$ of size $\left(N_{T} \times N_{R}\right)$ :

$$
\mathbf{x}_{u}=\mathbf{P}_{u} \mathbf{d}_{u}
$$

MC-CDMA signals of each user are created by a spreading code. Each data symbol spreads over the whole spreading code cycle. The entire spreading code cycle is transmitted simultaneously by assigning each chip code to the separate OFDM subcarrier.

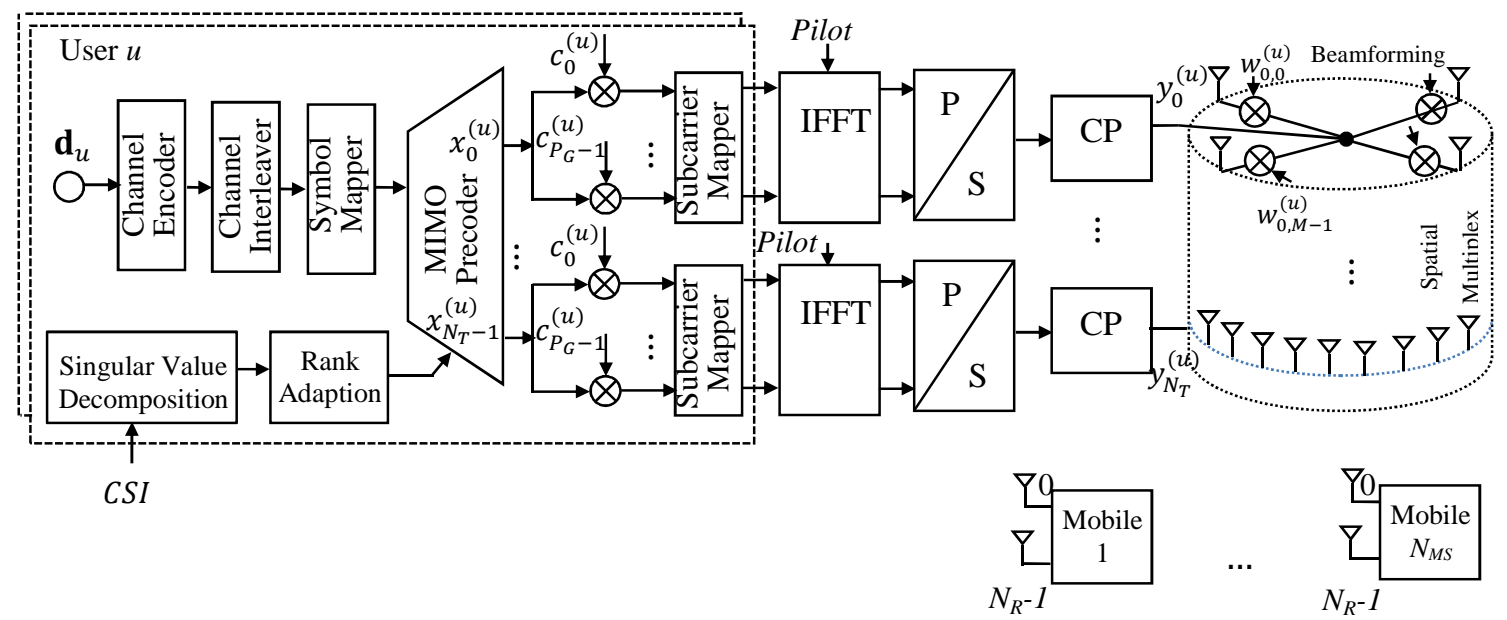

Figure 1. The proposed large-scale MIMO MC-CDMA model.

Without loss of generality, we consider typical MC-CDMA where the processing gain $P_{G}$ equals the number of the subcarriers $N_{C}$, that is $N_{C}=P_{G}$. Data symbols performed in the discrete time domain $i x^{u}[i]$ are copied into $N_{C}$ subcarriers, then multiply by the spreading code $\boldsymbol{c}_{u}=\left[c_{0}^{(u)}, c_{1}^{(u)}, \ldots, c_{P_{G}-1}^{(u)}\right]$ of user $u$. The MC-CDMA transmitted signal in transmitted signal 
vector at the discrete time $k \mathbf{y}_{u}[k]=\left[y_{0}^{(u)}[k], y_{1}^{(u)}[k], \ldots, y_{N_{T}-1}^{(u)}[k]\right]$ of user $u$, before beam forming $y_{n}^{(u)}[k]$ is of the form:

$$
y_{n}^{(u)}[k]=\frac{1}{\sqrt{N_{C}}} \sum_{m=0}^{N_{C}-1} \sum_{j=0}^{P_{G}-1} x_{n}^{(u)} c_{j}^{(u)} e^{j 2 \pi \frac{k m}{N_{C}}}
$$

\subsection{Multiple beamforming model}

\subsubsection{Transmit multiple beamforming}

In the system model, the paper proposes the implementation of multiple beamforming on each plane of cylindrical array antenna structure, that is, each individual mode is accompanied by eigen-beamforming The multiple beamforming corresponding to the own mode directly to MS will maximize SNR at the LOS direction and minimize at other directions combined with wideband modulation of the multicarrier method will be resistant to the frequency selective character of wideband channels, that is, channel response coefficients will be different at different frequencies. Once beamforming is done, spatial multiplexing implemented on modes corresponding to each lobe layer. Multiple beamforming can be performed in the frequency domain. That is, the transmitted signal vectors use DFT transformation to create vectors in the frequency domain. These vectors are then multiplied by the beamforming coefficients and use inverse IDFT to broadcast.

Overall, precoding using the multiple beamforming scheme is done by the sum of the weighted signals:

$$
s_{n}^{(u)}[k]=\sum_{m=0}^{M-1}\left(w_{n, m}^{(u)}\right)^{*} y_{n}^{(u)}[k]=\left(\mathbf{w}_{n}^{(\boldsymbol{u})}\right)^{H} \mathbf{y}_{u}[k]
$$

where $\mathbf{W}^{(\boldsymbol{u})}=\left[\mathbf{w}_{0}^{(\boldsymbol{u})}, \mathbf{w}_{1}^{(\boldsymbol{u})}, \ldots, \mathbf{w}_{N_{T}-1}^{(\boldsymbol{u})}\right]$ denotes beamforming coefficients of the $u$, each beamforming vector:

$$
\left(\mathbf{w}_{i}^{(\boldsymbol{u})}\right)^{H}=\left[\left(w_{i, 0}^{(u)}\right)^{*},\left(w_{i, 1}^{(u)}\right)^{*}, \ldots,\left(w_{i, M-1}^{(u)}\right)^{*}\right]=\left[\left(\mathbf{w}_{i}^{(\boldsymbol{u})}\right)^{T}\right]^{*}
$$

\subsubsection{Robustness optimization by Min-Max criteria}

The robustness optimizing problem is looking for the solution of beamforming weight vectors in order to minimize the worst case (the best in the worst condition in the set of received signals $\mathbf{r}_{f}$ (in the time domain or the frequency domain) and $M S E\left(\mathbf{r}_{f}, \hat{\mathbf{r}}_{f}\right)$ (mean square error) criteria, with constant $q>0$ and a $\mathbf{Q}$ positive define matrix [13]. The problem is stated by using Min-Max criteria as follows:

$$
\begin{gathered}
\mathbf{w}_{M N M}=\arg \min _{\mathbf{w}_{r}} \max _{\mathbf{r}_{f}: \mathbf{r}_{f}^{H} \mathbf{Q \mathbf { r } _ { f } \leq q ^ { 2 }}} \operatorname{MSE}\left(\mathbf{r}_{f}, \hat{\mathbf{r}}_{f}\right)=\arg \min _{\mathbf{w}_{r}} \max _{\mathbf{r}_{f}: \mathbf{r}_{f}^{H} \mathbf{Q r} \mathbf{r}_{f} \leq q^{2}} E\left\{\mid \hat{\mathbf{r}}_{f}-\right. \\
\left.\left.\mathbf{r}_{f}\right|^{2}\right\}=\arg \min _{\mathbf{w}_{r}} \max _{\mathbf{r}_{f}: \mathbf{r}_{f}^{H} \mathbf{Q} \mathbf{r}_{f} \leq q^{2}}\left\{\mathbf{w}_{r}^{H} \overline{\mathbf{R}}_{x} \mathbf{w}_{\boldsymbol{r}}+\left|\mathbf{r}_{f}\right|^{2}\left|1-\mathbf{w}_{r}^{H} \mathbf{A}_{r}\right|^{2}\right\}
\end{gathered}
$$

With the covariance matrix of the observation vector:

$$
\overline{\mathbf{R}}_{x}=E\left\{\left(\mathbf{r}_{f} \mathbf{r}_{f}^{H}\right)\right\}
$$

We have: 


$$
\begin{aligned}
& \overline{\mathbf{w}}_{M N M}=\arg \min _{\mathbf{w}_{r}} \max \quad \mathbf{r}_{f}: \mathbf{r}_{f}^{H} \mathbf{Q} \mathbf{r}_{f} \leq q^{2} \quad \operatorname{MSE}\left(\mathbf{r}_{f}, \hat{\mathbf{r}}_{f}\right) \\
& \overline{\mathbf{R}}_{x}: \sum \max \left\{\operatorname{tr}\left(\overline{\mathbf{R}}_{x} \overline{\mathbf{R}}_{x}^{H}\right)\right\}
\end{aligned}
$$

The solution of the equation are determined by using the Lagrange multiplier method [13]:

$$
\overline{\mathbf{w}}_{M N M}=q^{2} \frac{\overline{\mathbf{R}}_{x}^{-1} \mathbf{A}_{r}}{1+q^{2} \mathbf{A}_{r}^{H} \overline{\mathbf{R}}_{x}^{-1} \mathbf{A}_{r}}
$$

Approximate solution of the weight vectors can be found by the interactive methods including steepest descent algorithm, gradient method, conjugate LMS (Least Mean Squares) algorithm, and the recursive least squares algorithm [14].

\subsection{Channel model}

MIMO channel model is as follows:

$$
\left[\begin{array}{c}
r_{1} \\
r_{2} \\
\vdots \\
r_{N_{R}}
\end{array}\right]=\left[\begin{array}{cccc}
h_{1,1} & h_{1,2} & \cdots & h_{1, N_{T}} \\
h_{2,1} & h_{2,2} & \cdots & h_{2, N_{T}} \\
\vdots & \vdots & \ddots & \\
h_{N_{R}, 1} & h_{N_{R}, 2} & \cdots & h_{N_{R}, N_{T}}
\end{array}\right]\left[\begin{array}{c}
s_{1} \\
s_{2} \\
\vdots \\
s_{N_{T}}
\end{array}\right]+\left[\begin{array}{c}
n_{1} \\
n_{2} \\
\vdots \\
n_{N_{R}}
\end{array}\right]
$$

where $\mathbf{r}=\left(r_{1}, r_{2}, \ldots, r_{N_{R}}\right)^{T}$ is a set of signals received from $N_{R}$ receive antennas of mobile station. Because MIMO spatial multiplexing system takes advantage of transmit diversity in space over time caused by fading and multipath combined with signal orthogonalization. The signal detection in the receiver is sequence detection. Therefore, for sequence detection procedure, we set up signals and channels as follows: Suppose that data is divided into blocks including $K$ symbols. In each block, to avoid inter block interference, we insert $P$ vector zero containing $N_{T}$ elements and $N$ is the number of useful data samples with $K=N+P$. The channel is Finite Impulse Response fading channel (FIR) having $L$ multipath on each link from one transmit antenna to one receive antenna. Choose $P$ to satisfy $P \geq L-1$. Signal received at the $j^{\text {th }}$ receive antenna in discrete time domain is of the form:

$$
r_{j}[k]=\sum_{i=0}^{N_{T}-1} \sum_{l=0}^{L-1} h_{j, i}^{l} s_{i}[k-l]+n_{j}[k]
$$

where, $r_{j}[k]$ is the signal sample received at the $j^{\text {th }}$ antenna at the discrete time $k$ vector $\mathbf{r}[k]=$ $\left(r_{1}[k], r_{2}[k], \ldots, r_{N_{R}}[k]\right)^{T}$ is output vector at the time $k$ with $k=0,1, \ldots, K-1$ being elements of received vector $\mathbf{r}=\left(\mathbf{r}^{T}[0], \mathbf{r}^{T}[1], \ldots, \mathbf{r}^{T}[K-1]\right)^{T} ; h_{j, i}^{l}$ is the $l^{\text {th }}$ element of the channel response $\mathbf{h}_{j, i}$ where $l=0,1, \ldots L-1$; transmitted signal vector at time $k$ $\mathbf{s}[k]=\left(s_{1}[k], s_{2}[k], \ldots, s_{N_{T}}[k]\right)^{T}$; The noise that affects the received signal samples is $\mathbf{n}[k]=$ $\left(n_{1}[k], n_{2}[k], \ldots, n_{N_{R}}[k]\right)^{T}$; The transmitted signal vector $\mathbf{s}=\left(\mathbf{s}^{T}[0], \mathbf{s}^{T}[1], \ldots, \mathbf{s}^{T}[K-1]\right)^{T}$; The AWGN noise vector $\mathbf{n}=\left(\mathbf{n}^{T}[0], \mathbf{n}^{T}[1], \ldots, \mathbf{n}^{T}[K-1]\right)^{T}$.

Band Toeplitz channel matrix:

$$
\mathbf{H}=\left[\begin{array}{ccccc}
\mathbf{H}^{0} & \mathbf{0} & \ldots & \mathbf{0} \\
\vdots & \mathbf{H}^{0} & \ddots & \vdots \\
\mathbf{H}^{L-1} & \vdots & \ddots & \mathbf{0} \\
\mathbf{0} & \mathbf{H}^{L-1} & \ddots & \mathbf{H}^{0} \\
\vdots & \vdots & \ddots & \vdots \\
\mathbf{0} & \mathbf{0} & \ddots & \mathbf{H}^{L-1}
\end{array}\right]
$$

with 


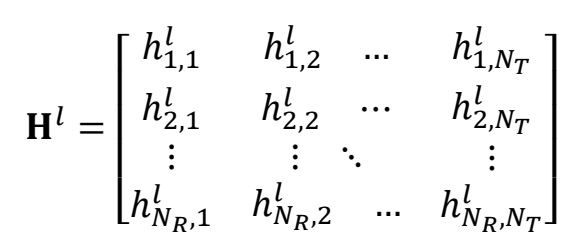

and

$$
\tilde{\boldsymbol{r}}=\widetilde{\mathbf{H}} \widetilde{\mathbf{s}}+\widetilde{\mathbf{n}}
$$

with

$$
\widetilde{\boldsymbol{r}}=\left[\begin{array}{c}
\mathfrak{R}\{\mathbf{r}\} \\
\mathfrak{I}\{\mathbf{r}\}
\end{array}\right], \widetilde{\mathbf{s}}=\left[\begin{array}{c}
\mathfrak{R}\{\mathbf{s}\} \\
\mathfrak{I}\{\mathbf{s}\}
\end{array}\right], \widetilde{\mathbf{H}}=\left[\begin{array}{cc}
\mathfrak{R}\{\mathbf{H}\} & -\mathfrak{I}\{\mathbf{H}\} \\
\mathfrak{I}\{\mathbf{H}\} & \mathfrak{R}\{\mathbf{H}\}
\end{array}\right], \widetilde{\mathbf{n}}=\left[\begin{array}{c}
\mathfrak{R}\{\mathbf{n}\} \\
\mathfrak{I}\{\mathbf{n}\}
\end{array}\right]
$$

where $\mathfrak{R}\{$.$\} denotes the real part and \mathfrak{I}\{$.$\} denotes the imaginary part of \{$.$\} .$

\section{MULTIUSER DETECTION}

Signals are received via MIMO equalizer as shown on Figure 2:

$$
\chi=\mathbf{r} \boldsymbol{\alpha}^{(u)^{T}}
$$

where $\boldsymbol{\alpha}^{(u)}=\left(\alpha_{0}^{(u)}, \alpha_{1}^{(u)}, \ldots \alpha_{N_{R}-1}^{(u)}\right)^{T}$ is a set of equalization complex weight coefficients of MIMO channels and selected by different criteria. Multi-user detection recovers user data based on utilizing the advantages of frequency diversity of multicarrier modulation. Subcarrier components $m=0,1, \ldots, N_{C}-1$ corresponding to the received data $d^{(u)}[i]$ are coherently detected by using FFT, then multiply by weight coefficients, $g_{u, m}$, to optimize power of received signals scattered in frequency domain due to fading and multiuser interference.

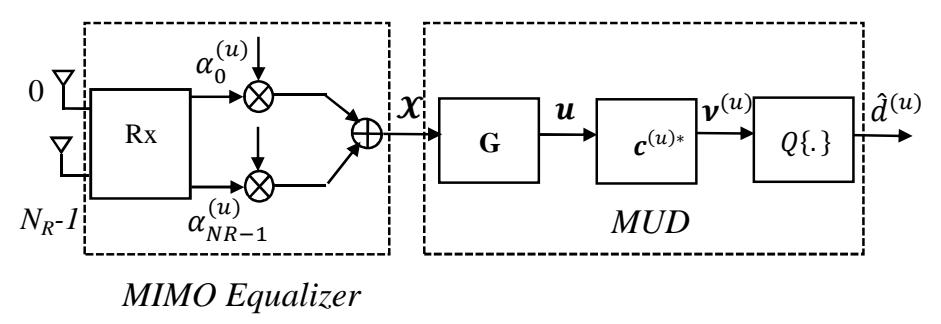

Figure 2. MIMO equalizer and MUD at the receiver.

Signals after the equalizing filter $\mathbf{G}$ :

$$
\mathbf{u}=\mathbf{G} \chi=\left(u_{0}, u_{1}, \ldots, u_{N_{C}-1}\right)^{T}
$$

with equalizing matrix:

$$
\mathbf{G}=\left[\begin{array}{ccc}
g_{0,0} & \cdots & \mathbf{0} \\
\vdots & g_{i, i} & \vdots \\
\mathbf{0} & \cdots & g_{N_{C}-1, N_{C}-1}
\end{array}\right]
$$

Signals after despreading:

$$
\mathbf{v}^{(u)}=\mathbf{c}^{(u) *} \mathbf{u}^{T}
$$

Data is derived from decision module:

$$
\hat{d}^{(u)}[k]=Q\left\{v^{(u)}[k]\right\}
$$


Multiuser detection techniques are applied to the proposed models and performed in simulation including [15]: (1) Zero Forcing (ZF); (2) Equal Gain Combining (EGC) and (3) Maximum Ratio Combining MRC.

\subsection{Equal Gain Combining (EGC)}

Sets of combined weight coefficients $\left\{\alpha_{i}^{(u)}\right\}$ and $\left\{g_{l, l}\right\}$ are chosen to compensate phase shift of MIMO channel to achieve the co-phase of MIMO channels and compensate phase shift of channel on the carriers:

$$
\begin{gathered}
g_{l, l}=\frac{h_{l, l}^{*}}{\left|h_{l, l}\right|} \quad l=0,1, \ldots, N_{C}-1 \\
\alpha_{i}^{(u)}=e^{-j \phi_{i}} \quad i=0,1, \ldots, N_{R}-1
\end{gathered}
$$

The magnitude of combined coefficients is equal and do not depend on SNR of transmission link. EGC weight coefficients are calculated by channel state information (CSI).

\subsection{Zero Forcing (ZF)}

This scheme uses channel inversion and reduces multiple access interference by restoring orthogonality between symbols. This scheme is also called Orthogonality Restoring Combining (ORC). The equalization coefficient is of the form:

$$
g_{l, l}=\frac{h_{l, l}^{*}}{\left|h_{l, l}\right|^{2}} \quad l=0,1, \ldots, N_{C}-1
$$

In order to eliminate the influence of interference suppression accompanied by weak amplitude subcarriers on the strongly amplified link. The decision is made based on the sum of base band elements of subcarriers having amplitude higher than detection threshold. The weight coefficients are chosen as following:

$$
g_{l, l}=\frac{h_{l, l}^{*}}{\left|h_{l, l}\right|^{2} u\left(\left|h_{l, l}\right|-\gamma\right)} \quad l=0,1, \ldots, N_{C}-1
$$

where $u($.$) is unit function and \gamma$ is detection threshold. Clearly, only noise values that are higher than optimal threshold $\gamma$ are amplified. With the given SRN ratio, the optimal threshold will be always available to achieve the minimum BER.

\subsection{Maximum Ratio Combining (MRC)}

MRC exploits MIMO spatial diversity to maximize SNR at the receiver, then minimize bit error probability. With given instant CSI, signals are combined in the quality of internal channels and phase synchronized. MIMO weight coefficients:

$$
\alpha_{i}^{(u)}=\frac{h_{i}^{*}}{\sigma_{i}^{2}}=\frac{\left|h_{i}\right| e^{-j \phi_{i}}}{\sigma_{i}^{2}} \quad i=0,1, \ldots, N_{R}-1
$$

MRC will combine synchronously signals of the other subcarriers by taking the weighted average of the subcarriers. The weight factor is the complex conjugate of channel coefficients corresponding the subcarriers, that is, the weight factors are selected by:

$$
g_{l, l}=h_{l, l}^{*} \quad l=0,1, \ldots, N_{C}-1
$$


With the selection of such weight coefficient values, MRC method has compensated the phase shift of channels and taken the weighted average value of signals after each filter to match coefficients proportional to the amplitude of the subcarriers.

\section{SIMULATION RESULTS}

The Monte-Carlo simulation is used to compute the system performance to verify the efficiency of the proposed system in different simulation conditions. The simulation is used to evaluate the performance of the proposed system and estimate the impact of parameters on the performance of the whole system. Parameters are composed of: Signal to Noise Ratio (SNR); the number of antennas $(M)$; the number of users $(K)$; multiple access interference. Bit error rate BER is used to define the performance of the system.

The Monte-Carlo simulation algorithm includes serial steps: Set up system configuration; create user data; MIMO precoding; create MC-CDMA transmit signals using OFDM; insert CP; create beamforming; receive signals; equalize MIMO; equalize MUD; demodulate, despread to recover data; compare with source data, calculate BER. The last estimate is calculated as the average of all $Q$ measured values after each simulation.

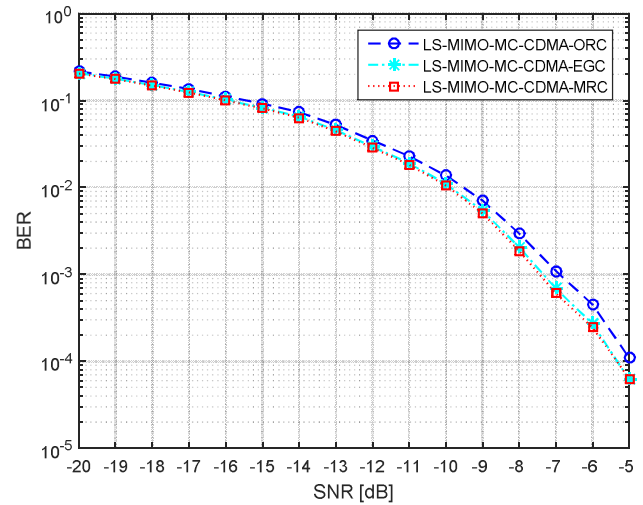

(a)

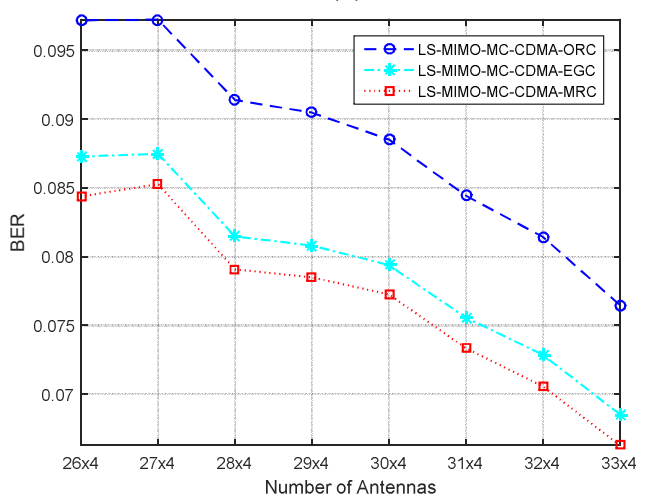

(c)

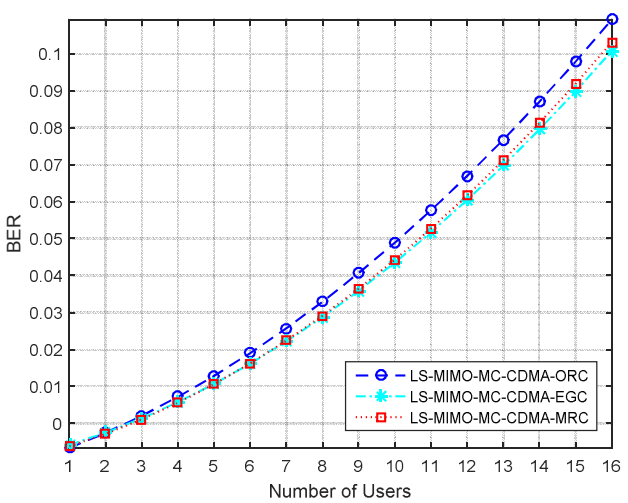

(b)

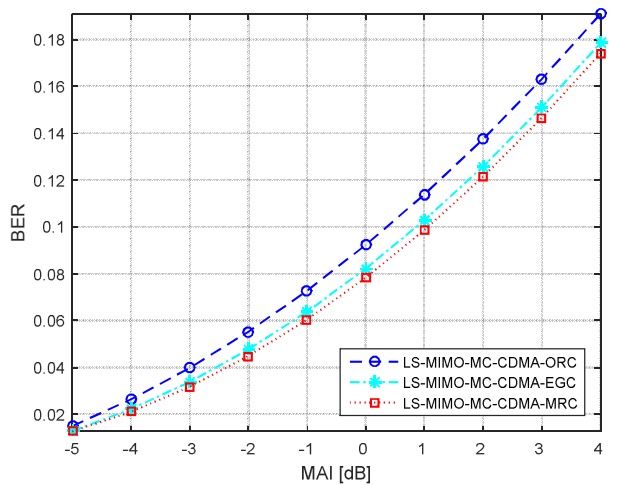

(d)

Figure 3. BER (a) SNR, (b) the number of the users, (c) the number of the antennas and (d) multiple access interference MAI.

Assuming that perfect CSI is available. Rayleigh fading channel used in the simulation has 10 multipaths [16]. The interference angle of incident is $-20^{\circ}$. AWGN noise $n(t)=\mathcal{N}(0,1)$ has 
the standard deviation standardized at 1 shown at all receive antennas. Carrier frequency $f_{c}=$ $5.25 \mathrm{GHz}$ [14], OFDM $N_{C}=1024$ subcarriers, the number of OFDM symbols in each frame is 32 , the gap between subcarriers $f_{d}=64 \mathrm{kHz}$. The Wash-Hadarmard spreading code has length of $L=1024$; chip rate $=100 \mathrm{Mchip} / \mathrm{s}$; sampling rate $=200 \mathrm{MHz}$. We use the URA array configuration including 128 elements, 32 MIMO channels, the subarray equals 4 .

In the first scenario, multiple access interference (MAI) and the number users $K$ are fixed and equal $0 \mathrm{~dB}$ and 6 user respectively, SNR changes between -20 and $-5 \mathrm{~dB}$, step size is $1 \mathrm{~dB}$ while in the second scenario, the number of users varies from 1 to 16 , SNR and MAI are fixed at $-10 \mathrm{~dB}$ and $0 \mathrm{~dB}$. The third scenario, the number of antennas varies while fixing SNR $=-10 \mathrm{~dB}$, the number of users $K=6, \mathrm{MAI}=0 \mathrm{~dB}$. The forth scenario varies MAI. Figure 3 shows simulation results respectively. The characteristics of the system can be seen clearly on the graphs. The performance of LS-MIMO-MC-CDMA-MRC gives the best result. When SNR is higher than $-5 \mathrm{~dB}$, BER of the system is lower than $10^{-4}$. When the number of users increases, BER tends to increase linear. Especially, when the number of antennas increases, BER reduces because narrow beamforming effect allows multiple access interference.

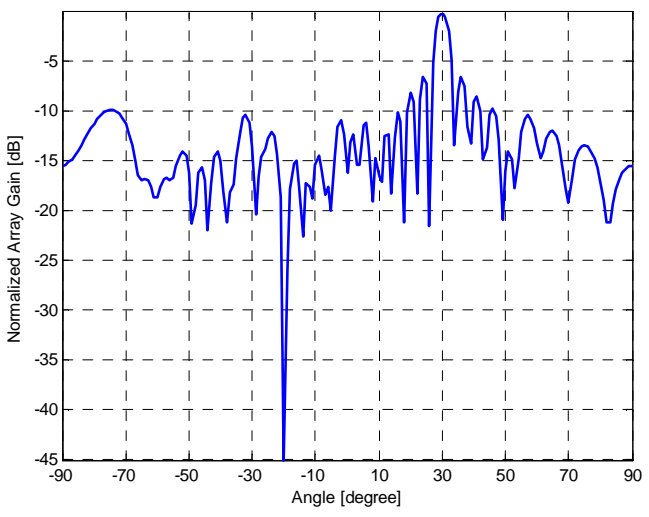

(a)

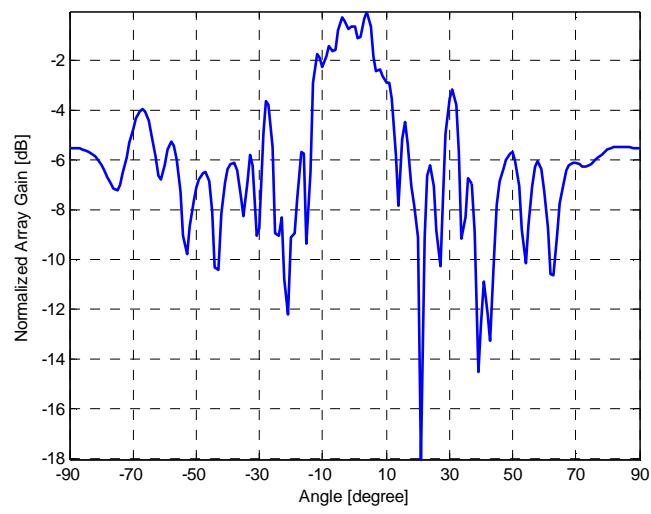

(c)

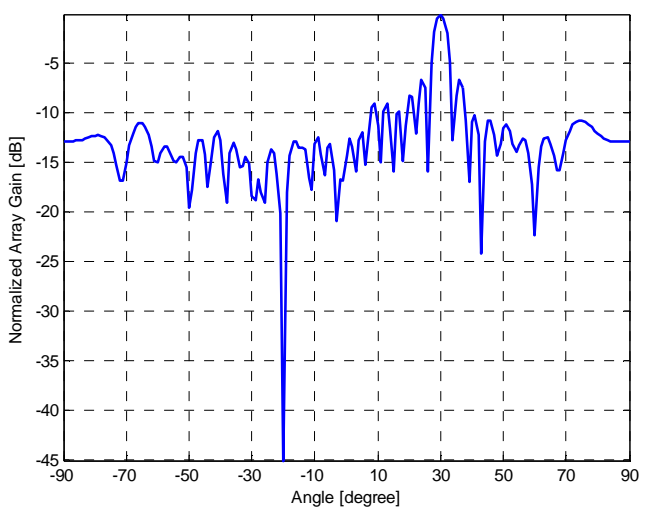

(b)

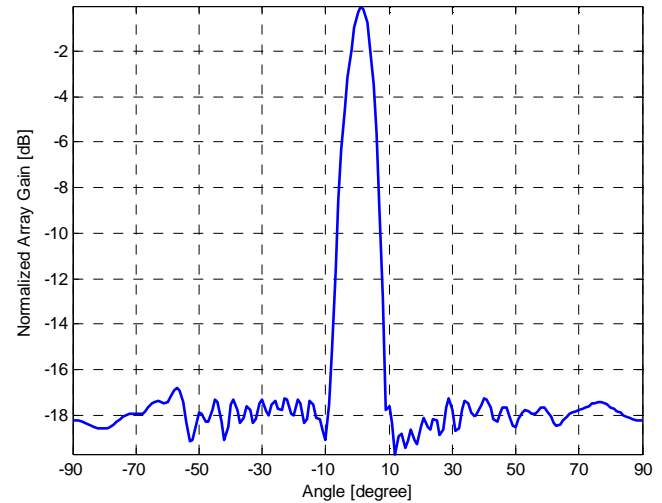

(d)

Figure 4. Beamforming patterns (a): MVDR algorithm; M=32x4-URA; DOA signal = 30 degree; DOA interference $=-20 \mathrm{SNR}=0 \mathrm{~dB}$; (b): LCMV algorithm; M=32x4-URA; DOA signal = 30 degree; DOA interference $=-20 \mathrm{SNR}=0 \mathrm{~dB}$; (c) Frost algorithm; $\mathrm{M}=32 \mathrm{x} 4-\mathrm{URA}$; DOA signal $=30$ degree; DOA interference $=-20 \mathrm{SNR}=0 \mathrm{~dB}$; and (d) Minimize the sub-beams by Min-Max criteria M=32x4-URA, DOA signal 0 degree; $\mathrm{SNR}=0 \mathrm{~dB}$. 
Beamforming patterns of beamforming algorithms under the influence of interference sources are shown in Figure 4. In Figure 4 (a), (b), (c) the response of the beamformer at the incident angle of interference is suppressed, while the response of the beamformer on Figure 4 (d) Min-max has very narrow beam to improve capacity and minimize multi user interference.

\section{CONCLUSIONS}

This paper has proposed a novel large scale Multi input - Multi output Multi carrier Code division multiple access (LS MIMO MC-CDMA) model applied to the Fifth-Generation Mobile Communication Systems (5G). This system uses combined cylindrical array antenna multiple beamforming architecture with spatial multiplexing. The model is optimized by Min-Max criteria in order to minimize side lobes and maximize the reduction of propagation loss. We apply reduced rank model to gain the efficiency in using transmission link as well as effectively utilizing the bandwidth. Beamforming by Min-Max optimization enhances the robustness of the systems in the domain having weak signals. In the next research, we determine the system capacity as well as the necessary and sufficient conditions so that this system can be controllable, observable in the case of moving users.

Acknowledgements. The authors would like to thank the Ministry of Science and Technology for the support under the project number NĐT.32.ITA/17.

\section{REFERENCES}

1. Elkashlan M., Duong T. Q., Chen H. H. - Millimeter-wave communications for 5G: fundamentals: Part I [Guest Editorial]," IEEE Communications Magazine 52 (9) (2014) $52-54$.

2. Qiao J., Shen X. S., Mark J. W., Shen Q., He Y., Lei L. - Enabling device-to-device communications in millimeter-wave 5G cellular networks, IEEE Communications Magazine 53 (1) (2015) 209-215.

3. Satoshi Suyama, Tasuki Okuyama, Yuki Inoue, Yoshihisa Kishiyama - 5G Multi-antenna technology, NTT Docomo Technical Journal 17 (4) (2015) 29-39.

4. Imai T., Kitao K., Tran N., et. al. - Study on Propagation Characteristics for Design of Fifth-Generation Mobile Communication Systems: Frequency Dependency of Path Loss in $800 \mathrm{MHz}$ to $37 \mathrm{GHz}$ Band in Small-Cell Environment, IEICE Technical report 114 (294) (2015) pp. 115-120.

5. Rappaport T.S. ; Sun S. ; Mayzus R. ; Zhao H.; Azar Y. ; Wang K.; Wong G.N. ; Schulz J.K. ; Samimi M.; Gutierrez F.- Millimeter wave mobile communications for 5G cellular: It will work!, IEEE Access 1 (2013) pp. 335-349.

6. Han S., Chih-Lin. I., Xu Z., Rowell C. - Large-scale antenna systems with hybrid analog and digital beamforming for millimeter wave 5G, IEEE Communications Magazine 53 (1) (2015) 186-194.

7. Ahmet Gokceoglu, Emil Björnson, Erik G. Larsson, Mikko Valkama - Spatio-Temporal Waveform Design for Multiuser Massive MIMO Downlink With 1-bit Receivers, IEEE Journal of Selected Topics in Signal Processing 11(2) (2017) 347-362. 
8. Sungwoo Park, Jeonghun Park, Ali Yazdan, Robert W. Heath - Exploiting Spatial Channel Covariance for Hybrid Precoding in Massive MIMO Systems, IEEE Transactions on Signal Processing 65 (14) (2017) pp. 3818-3832.

9. Kuang-Min Lin, Juinn-Horng Deng - Generalized side-information cancellation precoder design for multiuser MIMO downlink systems, IEEE International Symposium on Broadband Multimedia Systems and Broadcasting (BMSB) (2016) 1-5.

10. Elkashlan M., Duong T. Q., Chen H. H. - Millimeter-wave communications for 5G-Part 2: Applications, IEEE Communications Magazine 53 (1) (2015) 166-167.

11. Magueta R., Castanheira D., Rui S. and Gameiro D. - Non linear space-time equalizer for single-user hybrid mmWave massive MIMO systems, Proceed. 8th International Congress on Ultra Modern Telecommunications and Control Systems and Workshops (ICUMT), 2016.

12. Kyungchul Kim, Jungwoo Lee, and Huaping Liu - Spatial-Correlation-Based Antenna Grouping for MIMO Systems, IEEE Transactions on Vehicular Technology 59 (6) (2010) 2898-2905.

13. Yonina C. Eldar - Minimax MSE Estimation of Deterministic Parameters With Noise Covariance Uncertainties, IEEE Trans. Signal Processing 54 (1) (2006) 138-145.

14. Deyan P., Shouqiang D. and Jingjie J. - The smoothing Fletcher-Reeves conjugate gradient method for solving finite minimax problems, ScienceAsia 42 (2016) 40-45.

15. Kyung Seung Ahn - Performance analysis of MIMO-MRC system in the presence of multiple interferers and noise over Rayleigh fading channels, IEEE Transactions on Wireless Communications 8 (7) (2009) 3727-3735.

16. Mobile and wireless communications Enablers for the Twenty-twenty Information Society (METIS), Deliverable D1.4, METIS Channel Models, Document Number: ICT-317669METIS/D1.4. 